\title{
Potencial Miogênico Evocado Vestibular: Proposição de um Novo Instrumento
}

\section{Vestibular Evoked Myogenic Potential: We Propose a New Instrument}

\author{
Aline Cabral de Oliveira*, José Fernando Colafêmina **, Pedro de Lemos Menezes ***. \\ * Doutora em Ciências Médicas pela Faculdade de Medicina de Ribeirão Preto - USP. Professora da Universidade Federal de Sergipe. \\ *** Doutor em Ciências Médicas, Otorrinolaringologia. Professor Livre-docente da Faculdade de Medicina de Ribeirão Preto - USP. \\ **** Doutor em Física Aplicada à Medicina e Biologia. Professor Adjunto da Universidade Estadual de Ciências da Saúde de Alagoas. \\ Instituição: Departamento de Oftalmologia, Otorrinolaringologia e Cirurgia de Cabeça e Pescoço. Faculdade de Medicina de Ribeirão Preto - Universidade de São \\ Paulo (USP) e Laboratório Instrumentação e Acústica (LIA) Universidade Estadual de Ciências da Saúde de Alagoas (UNCISAL) \\ Maceió / AL - Brasil. \\ Endereço para correspondência: Prof. Dr. Pedro de Lemos Menezes - Rua Dr. Antônio Cansação, 55 - Apto. 703 - Ponta Verde - Maceió / AL - Brasil - CEP: \\ 57035-190 - Telefone: (+55 82) 3315-6813 - E-mail: pedrodelemosmenezes@gmail.com \\ Artigo recebido em 11 de Julho de 2010. Artigo aprovado em 22 de agosto de 2010.
}

\section{RESUMO}

Introdução:

Atualmente, ainda não existe um instrumento específico para a avaliação dos potencias miogênicos evocados vestibulares, sendo a mesma realizada por meio de um equipamento desenvolvido para a captação de potenciais evocados auditivos, disponível no mercado. Assim, as funções de amplificação, filtragem, estimulação, janela de captação, rejeição de artefatos e características de promediação, bem como o próprio software de gerenciamento, ficam muito a desejar para a nova função que lhe é atribuída.

Objetivo: Comparar latências e amplitudes das componentes p13 e n23, e os índices de assimetria, entre um equipamento considerado padrão-ouro (EP25), adaptado para o registro deste potencial, e o novo equipamento desenvolvido.

Método: Estudo experimental com 11 ouvintes normais. A partir de estímulos sonoros tone-burst de $10 \mathrm{~ms}$, frequência de $500 \mathrm{~Hz}$, com intensidade de 90 dBNAn, foram registrados potenciais miogênicos evocados com janelas de $80 \mathrm{~ms}$.

Resultados: Pode-se constatar ausência de diferenças significativas, quando comparados os dois instrumentos, quanto aos parâmetros de latência e amplitude, exceto para a latência de n23, com valor de $\mathrm{p}=0,005$. O valor do índice de assimetria inter-pico p13-n23, para o novo aparelho (20,1\%), foi significativamente menor que o encontrado para o EP25 (30,5\%), com p menor que 0,01.

Conclusão: Desta forma, conclui-se, que não existem diferenças para as latências e amplitudes do VEMP entre o EP25 e o novo dispositivo desenvolvido, apresentando, este último, maior proximidade com os valores descritos na literatura e menor índice de assimetria da amplitude inter-pico p13-n23. Forma de estudo: prospectivo, clínico e experimental. Identifier: NCT01132105

Palavras-chave: potenciais evocados, vestíbulo do labirinto, diagnóstico.

\section{SUMMARY}

Introduction:

Objective:

Method:

Results:

Conclusion:

Keywords:
Currently, there is still no specific instrument for assessment of vestibular evoked myogenic potentials, with the same performed by a device designed to capture auditory evoked potentials available in the market. Thus, the functions of amplification, filtering, stimulation, window capture, rejection of artifacts and features of averaging, as well as its management software, are much to be desired for the new role assigned to it.

To compare latencies and amplitudes of components p13 and n23, and the indices of asymmetry between a devices considered the gold standard (EP25), adapted to record the potential and the new equipment developed.

An experimental study with 11 normal hearing. From the tone-burst sound stimuli of $10 \mathrm{~ms}$, frequency of $500 \mathrm{~Hz}$ with intensity 90 dBNAn, evoked myogenic potentials were recorded with $80 \mathrm{~ms}$ windows. You can see no significant differences when comparing the two instruments regarding the parameters of latency and amplitude, except for the latency of $\mathrm{n} 23$, with $\mathrm{p}=0.005$. The value of asymmetry index inter-p13-n23 peak for the new unit (20.1\%) was significantly lower than that found for the EP25 (30.5\%), with p less than 0.01 .

Thus, we conclude that there are no differences for the latencies and amplitudes of VEMP between the EP25 and the new device developed by presenting the latter, closer to the values reported in literature and a lower index of asymmetry of amplitude between p13-n23 peak. Study form: Prospective clinical and experimental. Identifier: NCT01132105.

evoked potentials, vestibule, labyrinth, diagnosis. 


\section{INTRODUÇÃO}

O potencial miogênico evocado vestibular (VEMP) consiste em um reflexo vestíbulo-cervical, de média latência, decorrente da estimulação acústica, de forte intensidade, do sáculo (1-3). A atividade eletromiográfica dos músculos do pescoço, em especial do músculo esternocleidomastóideo, é captada por meio de eletrodos de superfície (4).

O reflexo vestibulocervical se origina no sáculo, é transmitido para os neurônios do Gânglio de Scarpa, caminha pelo nervo vestibular inferior, núcleo vestibulare tracto vestibuloespinhal e chega aos neurônios motores do músculo esternocleidomastóideo (5-7).

Este potencial investiga se o órgão vestibular é hipersensível ao som (causada por algumas patologias da orelha interna) e se existem alterações no sáculo, no nervo vestibular inferior ou no núcleo vestibular (8-14).

Atualmente, apesar do aumento nos estudos sobre VEMP (2), os trabalhos ainda são desenvolvidos com um instrumento adaptado, o que representa limitações quanto à investigação dos parâmetros do exame (15-28). O desenvolvimento de um aparelho para a avaliação dos potencias miogênicos evocados vestibulares, com funções específicas de amplificação, filtragem, estimulação e janela de captação, permitirá um ajuste minucioso e uma investigação mais aprofundada a respeito do assunto, o que, com um dispositivo adaptado que avalia potenciais evocados auditivos, não é possível.

Desta forma, o presente estudo teve o objetivo de comparar, através de um teste-piloto, as latências e amplitudes das componentes p13 e n23 e índices de assimetria, obtidos na aquisição do potencial miogênico evocado vestibular, entre um equipamento considerado padrãoouro, adaptado para o registro deste potencial, e o equipamento desenvolvido com fins específicos de avaliação do VEMP.

\section{Método}

O protocolo desta pesquisa está baseado na legislação pertinente, Resolução № 196/96, do Conselho Nacional de Saúde, do Ministério da Saúde, para estudos com seres humanos e foi aprovado pelo Comitê de Ética em Pesquisa da instituição onde a pesquisa foi realizada, com protocolo no 625 , no dia 01 de dezembro de 2006.

A amostra foi composta por 11 indivíduos, sendo seis do gênero feminino e cinco do gênero masculino, por demanda espontânea do Laboratório de Instrumentação e
Acústica - LIA, considerando-se os seguintes critérios de inclusão: Idades entre 18 e 35 anos e limiares auditivos iguais ou inferiores a 20 dBNA com diferenças entre as orelhas, por frequência, iguais ou inferiores a $10 \mathrm{~dB}$. Os critérios de exclusão adotados foram: Exposição a ruído ocupacional ou de lazer; cirurgias na orelha; mais de três infecções de orelha no ano corrente; uso de medicação ototóxica; presença de zumbido, vertigens, tonturas ou outras alterações cócleo-vestibulares; presença de alterações sistêmicas que possam contribuir para patologias cócleo-vestibulares, como diabetes, hipertensão arterial e dislipidemias e/ou alterações hormonais.

Inicialmente, foi aplicado um questionário de triagem, em seguida a pesquisa foi explicada verbalmente e um termo de consentimento livre e esclarecido foi entregue e assinado antes do início do experimento pelos sujeitos que aceitaram participar do estudo.

Antes da captação do VEMP, foram realizados os seguintes procedimentos para verificação da normalidade da audição: a) Otoscopia para a inspeção do meato acústico externo e da integridade da membrana timpânica com um otoscópio da marca Welch Allyn 29090, com espéculos esterilizados. b) Audiometria tonal liminar, com audiômetro da marca Interacoustic modelo AC40, com tom puro pulsátil, em cabine acústica.

O potencial miogênico evocado vestibular (VEMP) foi registrado em dois equipamentos, no equipamento padrão-ouro (EP25 da Interacoustic - instrumento amplamente utilizado para avaliação de potenciais de curta, média e longa latências) e no aparelho desenvolvido.

O novo aparelho foi desenvolvido no Laboratório de Instrumentação e Acústica (LIA) da UNCISAL e no Centro de Instrumentação Dosimetria e Radioproteção da FFCLRPUSP (CIDRA), o qual é composto por amplificadores biológicos, com ganho até 200.000x, filtros passa-banda configuráveis entre $010.000 \mathrm{~Hz}$, sistema de proteção elétrica e um sistema lógico que possibilita a investigação aprofundada do VEMP, uma vez que permite amplo controle dos parâmetros do exame, diferente dos modelos disponíveis no mercado que possuem parâmetros prédefinidos. Além disso, o aparelho possui interface com o computador pela placa de som, o que pode diminuir seu valor para comercialização em torno de $50 \%$, quando comparado aos modelos disponíveis atualmente. Em ambos os equipamentos, os estímulos sonoros foram apresentados por meio de fones de inserção ER-3A.

Inicialmente, os voluntários permaneceram sentados em uma cadeira e foi realizada limpeza da pele dos mesmos, utilizando-se pasta abrasiva, álcool e algodão. Em seguida, foram colocados eletrodos descartáveis do tipo 
prata e cloreto de prata $(\mathrm{Ag} / \mathrm{AgCl})$ para registro do potencial, após utilização prévia de pasta eletrolítica. O eletrodo ativo foi posicionado na metade superior do músculo esternocleidomastóideo, ipsilateral à estimulação; o eletrodo de referência, sobre a borda anterior da clavícula, ipsilateral, e o eletrodo terra, na linha média frontal.

Após a colocação dos eletrodos, procedeu-se com a avaliação da impedância entre os eletrodos não-inversor e o terra e entre os eletrodos inversor e o terra. Dessa forma, foi permitida impedância entre os eletrodos de até $3 \mathrm{k} \Omega$.

Para obtenção do registro dos potenciais miogênicos evocados vestibulares, no músculo esternocleidomastóideo, o paciente permaneceu sentando, com rotação lateral máxima de cabeça para o lado contralateral ao estímulo e manteve contração tônica do músculo em torno de $60 \mu \mathrm{V}$. O estímulo foi iniciado pela aferência direita e, posteriormente, repetido na aferência esquerda. As respostas foram replicadas, ou seja, registradas duas vezes do lado direito e duas vezes do lado esquerdo.

Na evocação do VEMP pelo equipamento EP25, foram promediados 200 estímulos do tipo tone burst, com duração de $10 \mathrm{~ms}$ (subida: 4, platô: 2, descida: 4), na frequência de $500 \mathrm{~Hz}$, apresentados em uma taxa de $5 \mathrm{~Hz}$, com intensidade de $90 \mathrm{~dB}$ NAn, utilizando-se um filtro passa banda de 5 a $1.200 \mathrm{~Hz}$. Os registros foram realizados em janelas de $80 \mathrm{~ms}$ e um canal de registro, uma vez que a captação dos estímulos e a evocação dos potenciais foi ipsilateral. $\mathrm{Na}$ evocação pelo novo instrumento, foram utilizados os mesmos parâmetros para o teste, com exceção, apenas para a filtragem, a qual foi de 5 a $2.200 \mathrm{~Hz}$. Os filtros utilizados foram diferentes, uma vez que, o equipamento padrão-ouro, semelhante aos equipamentos para captação de potenciais auditivos disponíveis no mercado, apresenta limitação quanto a essa configuração. Assim, como o novo equipamento apresenta uma ampla possibilidade de ajustes, foi utilizada a configuração de filtro mais adequada, de acordo com a literatura.

Para realizar a interpretação dos achados, as respostas foram analisadas por meio da morfologia, demarcandose as ondas p13 e n23, pelas latências dos primeiros picos negativo e positivo. Em seguida, foi obtido o índice de assimetria $(13,14)$, calculado pela equação 1 , que poderá ser recordada a seguir:

$$
\mathrm{R}(\%)=(\mathrm{AE}-\mathrm{AD} / \mathrm{AE}+\mathrm{AD}) \cdot 100
$$

Onde, $\mathrm{A}=$ amplitude de VEMP; e D e E em referência aos lados direito e esquerdo, respectivamente.

Os dados foram tabulados e processados pelo aplicativo para microcomputador Statistical Package for
Social Sciences (SPSS) versão 16.0. Para a descrição dos dados, fez-se uso da apresentação tabular e gráfica das médias, das medianas, dos desvios-padrão e de percentis. A normalidade das amostras foi observada através do teste de Kolmogorov-Smirnov e Shapiro-Wilk.

Após os dados obtidos serem caracterizados com a utilização de técnicas de estatística descritiva, aplicou-se o teste não-paramétrico de Wilcoxon para comparação das latências das componentes do VEMP (p13 e n23), evocadas pelos dois equipamentos. O teste de Qui-quadrado foi aplicado para a comparação das frequências obtidas pelo cálculo do índice de assimetria. Os valores foram considerados significativos para $p$ menor que $0,05(p<0,05)$. O valor do erro beta admitido foi de 0,1 .

\section{RESULTADOS}

A amostra estudada foi composta por 11 voluntários, sendo 5 do gênero masculino $(45,45 \%)$ e 6 do feminino (54,55\%). Verificou-se faixa etária entre 19 e 31 anos, média de idade de 21,18 anos e desvio-padrão de 3,37 anos.

A normalidade das amostras, quanto às latências e amplitudes das ondas p13 e n23, foi observada, utilizandose os testes de Shapiro-Wilk e Kolmogorov-Smirnov, entretanto, não foi verificada distribuição normal para as variáveis: amplitudes de p13 e n23 do lado direito, para o novo equipamento, e amplitude de n23, do lado esquerdo, para o aparelho padrão-ouro. Dessa forma, foram utilizados os testes não-paramétricos de Wilcoxon e Qui-quadrado, a depender de serem variáveis médias ou frequências, respectivamente.

Em todos os sujeitos, foram captadas as ondas p13 e n23 do VEMP, com morfologia adequada, por meio da estimulação e captação unilateral, independente do aparelho utilizado.

Comparando-se os dois equipamentos em estudo, sem diferenciar os lados direito e esquerdo, constataram-se latências absolutas mais tardias para o equipamento padrão-ouro (Tabela 1).

$\mathrm{Na}$ Tabela 2, podem ser verificadas maiores amplitudes absolutas (em módulo) para as ondas p13 e n23, evocadas no novo instrumento, quando comparado ao EP25.

Os resultados obtidos, quanto às latências e amplitudes, das médias obtidas entre as orelhas esquerda e direita, para os dois instrumentos, podem ser melhores visualizados no Gráfico 1. 
Tabela I. Medidas da latência para os componentes do VEMP evocados no novo aparelho e no aparelho padrão-ouro.

\begin{tabular}{|c|c|c|c|c|c|c|}
\hline \multirow{2}{*}{$\begin{array}{l}\text { Componentes } \\
\text { do VEMP }\end{array}$} & \multicolumn{2}{|c|}{ Média } & \multicolumn{2}{|c|}{ Mediana } & \multicolumn{2}{|c|}{ Desvio-padrão } \\
\hline & $\begin{array}{l}\text { Aparelho } \\
\text { Novo }\end{array}$ & $\begin{array}{l}\text { Aparelho } \\
\text { Padrão }\end{array}$ & $\begin{array}{l}\text { Aparelho } \\
\text { Novo }\end{array}$ & $\begin{array}{l}\text { Aparelho } \\
\text { Padrão }\end{array}$ & $\begin{array}{c}\text { Aparelho } \\
\text { Novo }\end{array}$ & $\begin{array}{l}\text { Aparelho } \\
\text { Padrão }\end{array}$ \\
\hline$p 13$ & 14,77 & 16,84 & 14,40 & 17,66 & 2,75 & 2,83 \\
\hline$n 23$ & 24,66 & 26,56 & 24,22 & 27,00 & 3,42 & 3,30 \\
\hline
\end{tabular}

* Valores de latência em milissegundos (ms)

Tabela 2. Medidas de amplitude para os componentes do VEMP evocados no novo aparelho e no aparelho padrão-ouro.

\begin{tabular}{lcccccc}
\hline $\begin{array}{l}\text { Componentes } \\
\text { doVEMP }\end{array}$ & \multicolumn{2}{c}{ Média } & \multicolumn{2}{c}{ Mediana } & \multicolumn{2}{c}{ Desvio-padrão } \\
& $\begin{array}{c}\text { Aparelho } \\
\text { Novo }\end{array}$ & $\begin{array}{c}\text { Aparelho } \\
\text { Padrão }\end{array}$ & $\begin{array}{c}\text { Aparelho } \\
\text { Novo }\end{array}$ & $\begin{array}{c}\text { Aparelho } \\
\text { Padrão }\end{array}$ & $\begin{array}{c}\text { Aparelho } \\
\text { Novo }\end{array}$ & $\begin{array}{c}\text { Aparelho } \\
\text { Padrão }\end{array}$ \\
\hline p13 & 13,38 & 10,42 & 11,62 & 9,59 & 16,56 & 11,09 \\
n23 & 14,70 & 14,05 & 13,28 & 9,72 & 14,09 & 14,18 \\
\hline
\end{tabular}

* Valores (módulo) de amplitude em microvolts $(\mu \mathrm{V})$

Aplicando-se o teste de Wilcoxon, pode-se constatar ausência de diferenças estatisticamente significativas, quando comparados os dois instrumentos quanto aos parâmetros de latência e amplitude, dos componentes p13 e n23, exceto para a latência de n23, onde observou-se diferença significativa com valor de $\mathrm{p}=0,005$.

Fazendo uso da fórmula para cálculo do índice de assimetria $(13,14)$, em sujeitos normais, pode-se observar maiores valores de assimetria para o aparelho padrão-ouro $(22,6 \%)$ que para o novo instrumento $(17,3 \%)$, no que se refere a componente $\mathrm{p} 13$. Quanto a componente $\mathrm{n} 23$, esses índices foram maiores na evocação pelo novo equipamento $(10,1 \%)$, contra $8,1 \%$ do padrão-ouro.

O teste Qui-quadrado, entretanto, não revelou diferenças estatisticamente significativas, entre o novo equipamento e o padrão ouro de mercado (EP25), quando comparados os índices de assimetria das amplitudes de p13 e n23, com valores de $p=0,14$ e 0,60 , respectivamente.

Nesse estudo, o valor do índice de assimetria interpico p13-n23, para o novo aparelho (20,1\%), foi significativamente menor que o encontrado para o EP25 (30,5\%), com p menor que 0,01 , utilizando-se também o teste Quiquadrado.

\section{DISCUSSÃO}

Escolheram-se estímulos tone burst, na frequência de $500 \mathrm{~Hz}$, uma vez que eles são mais efetivos que os clicks, para a obtenção do VEMP, e, dentre os estímulos tone burst, as baixas frequências $(\leq 1.000 \mathrm{HZ})$ são mais

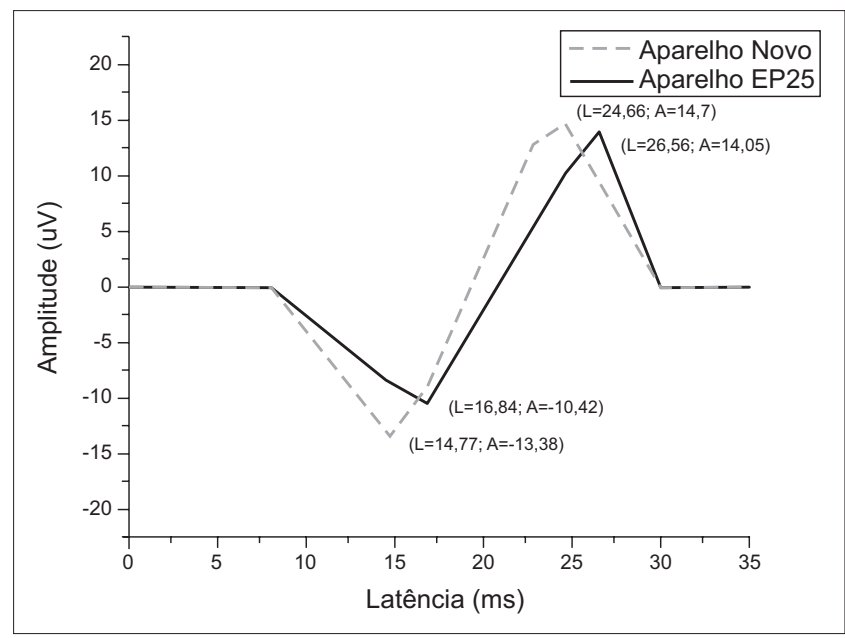

Gráfico 1. Valores médios de latência e amplitude para os componentes do VEMP evocados no novo aparelho e no aparelho padrão-ouro.

efetivas que as altas $(4,14)$, sendo, a de $500 \mathrm{~Hz}$, a mais utilizada $(7,14)$.

Quanto à intensidade do estímulo, foi escolhido 90 dBNAn, uma vez que, a maioria dos estudos, utiliza intensidades de estímulos iguais ou superiores esta intensidade (15-18).

Foram relatados diversos músculos para a captação do VEMP (17, 19-21). Nesse trabalho, utilizou-se o músculo ECM, uma vez que é, atualmente, o mais utilizado (14, 16, 22). Somado a isto, comparações de resultados do VEMP, no músculo ECM e no músculo trapézio, revelam que as respostas no ECM são mais homogênias (13). 
Em relação à nomenclatura das ondas do VEMP, $\mathrm{O}$ primeiro pico pode apresentar as seguintes denominações: A, p13, p14 e I. O segundo pico é referido na literatura como n23, n21 ou II $(7,24)$. Nesse estudo, optouse por utilizar as nomenclaturas para o primeiro e segundo picos, respectivamente, p13 e n23, uma vez que são as normalmente utilizadas pela literatura específica. A diferença entre as latências e amplitudes de n23 e p13 foi denominada de inter-pico, configurando a expressão da atividade elétrica muscular reflexa à estimulação sonora da mácula sacular.

No presente estudo, foram analisadas apenas as ondas p13 e n23, uma vez que as ondas mais tardias não estão presentes em todos os indivíduos normais e, portanto, não devem ser levadas em consideração para a interpretação do VEMP (13).

A observação da latência absoluta das respostas é importante para a identificação dos picos no traçado e para a observação de problemas que interfiram na velocidade de condução neural (13), além de ser um parâmetro reprodutível da resposta de VEMP, que independe da intensidade do estímulo e do nível de tensão eletromiográfica (1).

Em estudo realizado com 64 sujeitos normais (22), utilizando-se a mesma metodologia de teste para a evocação do VEMP, foram determinados valores de normalidade, para latência da onda p13, de até $20,3 \mathrm{~ms}$, e de $28,0 \mathrm{~ms}$, para n23. Entretanto, em outro trabalho (1), os valores de normalidade, para a onda p13, oscilam entre 13,8 e 18,5 ms; enquanto, n23 apresenta variação de 20,6 a 27,4 ms. Dessa forma, independente do parâmetro de normalidade e do equipamento utilizado (padrão-ouro de mercado ou novo aparelho desenvolvido), as latências absolutas, dos dois componentes do VEMP, se encontravam de acordo com os padrões de normalidade. Entretanto, foi necessário maior tempo de registro para serem captadas, cada uma das duas ondas, no aparelho padrão-ouro.

Os resultados encontrados, nesse trabalho, demonstram que, apesar de não existirem diferenças estatisticamente significativas, em relação à latência absoluta, entre os dois instrumentos utilizados, podem ser encontrados valores de latência mais precoces no novo instrumento, provavelmente, decorrente da utilização de filtros mais específicos, para a captação deste potencial.

Assim, as latências absolutas são consideradas parâmetros clínicos úteis, para a avaliação da condução neural, contribuindo no diagnóstico auxiliar de patologias neurológicas, como a esclerose múltipla, por exemplo (13).

Os valores absolutos de amplitude, encontrados nesse estudo, foram bastante variáveis, entre os indivíduos, e no mesmo sujeito, quando captados em momentos distintos, nos dois equipamentos (padrão-ouro e novo instrumento). Esses achados corroboram com a maioria dos estudos sobre VEMP (7, 22, 25-27).

Com a evocação do VEMP nos dois equipamentos, foram encontrados maiores valores de amplitudes absolutas no novo instrumento. Esses achados configuram aumento quantitativo na atividade do reflexo vestíbulocervical (28). Além disso, valores de desvios-padrão foram, frequentemente, menores no novo aparelho, o que retrata diminuição das variações nas amplitudes do VEMP, evocadas por este instrumento.

Mesmo assim, diversos autores concordam que, os valores de amplitude absoluta não devem ser utilizados na análise desse teste, uma vez que não podem ser reprodutíveis, em decorrência da grande variação intersujeito, e são dependentes de alguns fatores, como intensidade do estímulo e nível de contração tônica do músculo ECM (7, 22, 25-28).

Além disso, observaram-se valores absolutos de amplitude, para os dois equipamentos, muito diferentes do encontrado em alguns estudos que usaram a mesma metodologia de teste, o que pode ser justificado pela utilização de diferentes ganhos do amplificador. Assim, o ganho seria mais um fator interferente para a amplitude, o que reforça, ainda mais, a não utilização da amplitude absoluta, na análise do VEMP.

$\mathrm{Na}$ tentativa de anular esses efeitos interferentes e tornar a amplitude um parâmetro analisável no teste de VEMP, foi utilizado o índice de assimetria $(13,14)$. Com isso, por meio dessa equação, pode-se observar resultados considerados normais, para as componentes p13 e n23, segundo estudo anterior (14), o qual relata que eles devem ser inferiores a 34\%. Além disso, foi comprovada, estatisticamente, a ausência de assimetrias para essas ondas, captadas nos dois equipamentos.

Outra análise do VEMP que foi realizada, no presente estudo, considerando o índice de assimetria das amplitudes inter-picos p13-n23, revelou índices bem menores para o novo aparelho $(20,1 \%)$ que para o aparelho padrãoouro $(30,5 \%)$ e também para o encontrado em estudo anterior, $45,00 \%$ (13). Isso mostra que o equipamento desenvolvido tem maior efetividade na captação do VEMP que o padrão de mercado.

Assim, para a comparação inter-pessoal da amplitude das respostas, não devem ser utilizados os valores absolutos dessas amplitudes, mas sim, o índice de assimetria, visto que ele reflete a diferença interaural da amplitude, ponderada pela amplitude média dessa resposta. 


\section{CONCLUSÃO}

Não existem diferenças para as latências e amplitudes do VEMP entre o EP25 e o novo dispositivo desenvolvido, apresentando, este último, maior proximidade com os valores descritos na literatura e menor índice de assimetria da amplitude inter-pico p13-n23.

\section{REFERÊNCIAS BIBLIOGRÁFICAS}

1. Damen MMJ. Vestibular evoked myogenic potential (VEMP). Clinical application of the threshold. Medical Engineering. Eindhoven: Technische Universiteit Eindhoven; 2007.

2. Rauch RD. Vestibular evoked myogenic potencials. Otol Neurotol. 2006, 14:299-304.

3. Sazgar AA, Akrami K, Akrami S, Yazdi ARK. Recording of vestibular evoked myogenic potencials. Acta Med Iran. 2006, 44(1):13-6.

4. Akin F, Murnane O, Panus P, Caruthers S, Wilkinson A, Proffitt $\mathrm{T}$. The influence of voluntary tonic EMG level on the vestibular-evoked myogenic potencial. JRRD. 2004, 41(3B):473-80.

5. Sheykholeslami K, Kaga K. The otolithic organ as a receptor of vestibular hearing revealed by vestibular-evoked myogenic potentials in patients with inner ear anomalies. Hear Res. 2002, 165:62-7.

6. Rosengren SM, Colebatch JG. Vestibular evoked potencials (VsEPs) in patients with severe to profund bilateral hearing loss. Clin Neuroph. 2006, 117:1145-53.

7. Hall J. New handbook of auditory evoked responses. New York: Pearson Education; 2006.

8. Guillén VP, García EG, Piñero AG, Rey APD, Pérez CM. Potencial vestibular miogénico evocado: un aporte al conocimiento de la fisiología y patología vestibular: Patrones cuantitativos en la población normal. Acta Otorrinolaringol Esp. 2005, 56:349-53.

9. Sazgar A, Dortjai V, Akrami K, Akrami S, Yazdi AK. Saccular damage in patients with high-frequency sensorioneural hearing loss. Eur Arch Otorhinolaryngol. 2006, 263:60813.

10. Takeich N, Sakamoto T, Fukuda S, Inuyama Y. Vestibular evoked myogenic potencial (VEMP) in patients with acoustic neuromas. Auris Nasus Lar. 2001, 28:839-41.
11. Pollak L, Kushnir M, Stryjer R. Diagnostic value of vestibular evoked myogenic potencials in cerebellar and lower-brainstem stroke. Neuroph Clin. 2006, 36:227-33.

12. Young Y. Vestibular-evoked myogenic potencials: optimal stimulation and clinical application. Jour of Biom Sci. 2006, 13:745-51.

13. Almeida RR. Potenciais evocados miogênicos vestibulares: Estudo em indivíduos normais. São Paulo: Universidade de São Paulo; 1999.

14. Murofushi T, Matsuzaki M, Wu C. Short tone burst-evoked myogenic potencials on the sternocleidomastoid muscle: are these potentials also of vestibular origin?. Arch Otolaryngol Head Neck Surg. 1999, 125:660-4.

15. Magliulo G, Cuiuli G, Gagliardi M, Ciniglio-Appiani G, DAmico R. Vestibular evoked myogenic potentials ang glycerol testing. Laryngoscope. 2004, 114:338-42.

16. Colebatch JG, Halmagyi GM, Skuse NF. Myogenic potencials generated by a click-evoked vestibulocollic reflex. Neurol Neurosurg. Psychiatry. 1994, 57:190-7.

17. Ferber-Viart C, Duclaux R, Colleaux B, Dubreuil C. Myogenic vestibular-evoked potentials in normal subjects: A comparison between responses obtained from sternomastoid and trapezius muscles. Acta Otolaryngol (Stockh). 1997, 117:472-81.

18. Ribeiro S, Almeida RR, Caovilla HH, Ganança MM. Dos potenciais evocados miogênicos vestibulares nas orelhas comprometida e assintomática na Doença de Meniére unilateral. Rev Bras Otorrinolaringol. 2005, 77(1):60-6.

19. Wu C, Young Y, Murofushi T. Tone burst-evoked myogenic potencials in human neck flexor and extensor. Acta Otolaryngol (Stockh). 1999, 119:741-4.

20. Sakakura K, Takahashi K, Takayasu Y, Chikamatsu K, Furuya N. Novel method for recording vestibular evoked myogenic potential: Minimally invasive recording on neck extensor muscles. Laryngoscope. 2005, 115:1768-73.

21. Cody D, Bickford R. Averaged evoked myogenic responses in normal man Laryngoscope. 1969, 79:400-16.

22. Basta D, Todt I, Ernst A. Normative data for P1/N1 latencies of vestibular evoked myogenic potencials induced by air- or bone-conducted tone bursts. Clin Neuroph. 2005, 116:2216-9.

23. Welgampola M, Colebatch J. Characteristics and clinical 
applications of vestibular-evoked myogenic potencials. Neurol. 2005, 64:1682-8.

24. Murofushi T, Iwasaki S, Ozeki H, Ushio M, Chihara Y. Tone burst-galvanic ratio of vestibular evoked myogenic potential amplitudes: A new parameter of vestibular evoked myogenic potential? Clin Neuroph. 2007, 118:1685-90.

25. Ochi K, Ohashi T. Age-related changes in the vestibularevoked myogenic potencials. Otolaryngol Head Neck Surg. 2003, 129:655-9.
26. Su H, Huang T, Young Y, Cheng P. Aging effect on vestibular evoked myogenic potencial. Otol Neurotol. 2004, 25:977-80.

27. Ochi K, Ohashi T, Nishino H. Variance of vestibularevoked myogenic potencials. Laryngoscope. 2001, 111:522-7.

28. Lim CL, Clouston P, Sheean G, Yiannikas C. The influency of voluntary EMG activity and click evoked myogenic potencial. Muscle Nerve. 1995, 18:1210-3. 